\title{
The Bhattacharyya distance: enriching the P-box in stochastic sensitivity analysis
}

\author{
Sifeng $\mathrm{Bi}^{*}{ }^{, a}$, Matteo Broggi ${ }^{a}$, Pengfei Wei ${ }^{a, b}$, Michael Beer ${ }^{a, c, d}$ \\ ${ }^{a}$ Institute for Risk and Reliability, Leibniz Universität Hannover, 30167 Hannover, Germany \\ ${ }^{b}$ Civil Engineering and Architecture, Northwestern Polytechnical University, School of Mechanics, 710072 Xi'an, China \\ ${ }^{c}$ Institute for Risk and Uncertainty, University of Liverpool, L69 7ZF Liverpool, United Kingdom \\ ${ }^{d}$ International Joint Research Center for Engineering Reliability and Stochastic Mechanics, Tongji University, 200092 Shanghai, \\ China
}

1 Abstract: The tendency of uncertainty analysis has promoted the transformation of sensitivity analysis 2 from the deterministic sense to the stochastic sense. This work proposes a stochastic sensitivity analysis 3 framework using the Bhattacharyya distance as a novel uncertainty quantification metric. The 4 Bhattacharyya distance is utilised to provide a quantitative description of the P-box in a two-level procedure 5 for both aleatory and epistemic uncertainties. In the first level, the aleatory uncertainty is quantified by a 6 Monte Carlo process within the probability space of the cumulative distribution function. For each sample of the Monte Carlo simulation, the second level is performed to propagate the epistemic uncertainty by 8 solving an optimisation problem. Subsequently, three sensitivity indices are defined based on the 9 Bhattacharyya distance, making it possible to rank the significance of the parameters according to the reduction and dispersion of the uncertainty space of the system outputs. A tutorial case study is provided in the first part of the example to give a clear understanding of the principle of the approach with reproducible results. The second case study is the NASA Langley challenge problem, which demonstrates the feasibility of the proposed approach, as well as the Bhattacharyya distance metric, in solving such a large-scale, strongnonlinear, and complex problem.

15 Keywords: sensitivity analysis; uncertainty quantification, uncertainty propagation; Bhattacharyya distance; probability box

\section{Introduction}

As uncertainty treatment in model Verification and Validation (V\&V) becomes increasingly popular, the Bhattacharyya distance has been investigated as a promising Uncertainty Quantification (UQ) metric in stochastic model updating [1]. However, the deterministic methodologies are still widely used in practical engineering, and the Euclidian distance is probably the most common metric providing a geometric distance between two single data points. Alternatively, the Bhattacharyya distance is a stochastic metric between two sets of samples considering their probability distributions. By capturing uncertainty sources from both experimental and numerical data, the Bhattacharyya distance has been investigated as a more comprehensive UQ metric [2]. Nevertheless, its application in V\&V has been quite limited so far in both academic and engineering fields. Upon to the authors' previous work on the role of the Bhattacharyya distance in stochastic model updating [1], the main objective of this work is to further promote the

* Corresponding author: sifeng.bi@irz.uni-hannover.de (S. Bi) 
application of the Bhattacharyya distance in stochastic sensitivity analysis (SA) within a two-level procedure for uncertainty propagation and quantification.

Besides the stochastic model updating [3], the stochastic SA is another key component of $\mathrm{V} \& \mathrm{~V}$, generally performed prior to model updating, with the purpose to evaluate and rank the significance of the input parameters according to the system outputs [4]. One of the classical SA techniques is the global sensitivity based on Sobol's indices [5,6]. However, as a variance-based approach, it cannot be directly applied to applications where the parameters are described as imprecise random variables due to both aleatory and epistemic uncertainties [7]. Despite the recent development on SA techniques (e.g. polynomial expansion [8], covariance decomposition [9], Bayesian approach [10], analysis of variance [11], etc.), an extension based on the imprecise probability theory [12-14] is required to achieve a deeper understanding on both aleatory and epistemic uncertainties' contributions to the uncertainty of the system outputs [15]. Uncertainties in simulation and experiment processes can be divided into three sources:

- Uncertainties in parameterisation. The input parameters of the numerical model are imprecisely determined, such as the materials properties of novel composites, geometry sizes of complex structures, and random boundary conditions lead by the winds or earthquakes.

- Uncertainties in modelling. The numerical model always contains simplifications and approximations, such as the linearization of nonlinear behaviours, the hypothesis of frictionless joints, and the simplification of complex connections.

- Uncertainties in experiments. The measurements are driven by hard-to-control randomnesses, such as environment noise, measurement system errors, and human subjective judgements. More explanations of experimental uncertainty can be found in Ref. [16].

Due to the above unavoidable uncertainty sources, this work focuses on the implementation of a stochastic SA approach in which the Bhattacharyya distance can be embedded as a comprehensive and convenient UQ metric by capturing various uncertainty sources. In this work, the input model parameters are no longer treated as unknown-but-fixed constants, but investigated as four categories according to the involvement of aleatory uncertainty (natural variation) or/and epistemic uncertainty (lack of knowledge):

I) Parameters without any uncertainty, appearing as explicit constants;

II) Parameters with only epistemic uncertainty, appearing as unknown-but-fixed constants, bounded by a given interval;

III) Parameters with only aleatory uncertainty, appearing as random variables with fully determined probability characteristics such as distribution type, mean, variance, etc.;

IV) Parameters with both aleatory and epistemic uncertainties, appearing as imprecise probability variables with only vaguely determined uncertainty characteristics.

The above parameter categorisation is critical in this work, since different parameter categories have different representations of their uncertainty characteristics, and thus requiring different treatment for uncertainty quantification and propagation. To represent the uncertainty space of variables with imprecise probability, the Probability box (generally known as P-box) is proposed by Ferson et al. [17] as a graphic representation, which has been widely used in uncertainty analysis [18-20]. In the following context, the 
term "uncertainty space" is utilised as a general expression of the imprecise probability measure of an uncertain variable. In the typical probability theory, the probability space is defined as the mathematic triplet $(\Omega, \mathcal{F}, \mathbb{P})$, where $\Omega$ is the complete event space, $\mathcal{F}$ is the $\sigma$-algebra space of all possible events, $\mathbb{P}$ is the probability measure of the event. The probability space is based on the assumption that the probability measure $\mathbb{P}$ is precisely known, implying only the aleatory uncertainty is considered. In the context of imprecise probability, however, the aleatory and epistemic uncertainties can occur simultaneously. Hence, the uncertainty space herein can be seen as an extension of the precise probability space. More detailed information of the uncertainty space (i.e. imprecise probability space) and P-box can be referred to Ref. [18].

The application of P-boxes in SA has been developed, e.g., by Ferson et al. [21] via the Probability Bounds Analysis (PBA), which is further developed by Alvarez [22] in the non-specificity SA approach. However, the P-box still requires a quantitative measure to define an explicit ranking index in SA. Meanwhile, a further investigation of the P-box is required to differentiate the effects of the aleatory and epistemic uncertainties. In this work, the proposed stochastic SA approach is constructed within a two-level framework to quantify the aleatory and epistemic uncertainties, along the vertical and horizontal directions of the P-box. The Monte Carlo simulation and optimisation techniques are respectively utilised in these two levels to quantify and propagate uncertainties from the system inputs to outputs. The Bhattacharyya distance acts as the core of the SA framework by providing a quantitative measure of the P-box, which is the foundation for the proposed sensitivity indices. The three indices, namely the proportional index, the variance-based index, and the comprehensive index, are utilised to rank the significance of the parameters according to the reduction and dispersion characteristics of the output uncertainty space.

Two case studies are proposed to demonstrate the feasibility of the overall two-level approach, as well as the Bhattacharyya distance metric. The first case study, investigating the Ishigami function with an explicit formulation, allows the readers to achieve a better understanding of the principle of the approach with reproducible results. The second case study solves the SA task of the NASA UQ challenge problem [23]. Results of the current work are compared with previously published works $[7,24,25]$ to demonstrate the Bhattacharyya distance as a competent UQ metric in the proposed stochastic SA approach.

The novelties of this work involve three aspects. First, the Bhattacharyya distance is proposed as a novel UQ metric, which can be conveniently embedded into the overall SA framework to provide a quantitative measure of the P-box (i.e. the uncertainty space). Second, this work performs the stochastic SA in the background of uncertainty treatment, and more specifically, to investigate the significance of an input according to how much the uncertainty space of the output can be changed, when the uncertainty space of this input is reduced. Third, the proposed two-level approach provides a clear logic to differentiate the contributions of the aleatory and epistemic uncertainties to the uncertainty space of the output, by investigating the P-box along two directions in the two levels, respectively.

103 The following parts of this paper are organised as follows. Section 2 investigates the typical P-box from a novel viewpoint: the aleatory and epistemic uncertainty representations along the vertical and horizontal directions of the P-box, respectively. Section 3 presents the two-level procedure for uncertainty propagation 
104

105

106

107

108

109

110

111

112

113

114

115

116

117

118

119

120

121

122

123

124

125

126

127

128

129

130

131

132

133

134

135

136

137

and quantification, where the Monte Carlo simulation is performed in the first level for aleatory uncertainty propagation, and an optimisation is performed in the second level for epistemic uncertainty quantification. Section 4 explains how to embed the Bhattacharyya distance into the two-level procedure, and subsequently, three sensitivity indices are defined based on the Bhattacharyya distance. The variation of the results and calculation cost are also discussed in this section. The Ishigami function and the NASA UQ challenge problem are investigated in Sections 5 and 6, respectively. Section 7 gives the conclusions and perspectives.

\section{P-box representation of the imprecise probability uncertainty space}

The P-box provides a clear graphic representation of the uncertainty space of an imprecise random variable. Since the stochastic SA approach in this work is proposed in the background of imprecise probability, the distributional P-box is investigated in this section. A distributional P-box is essentially a family of Cumulative Distribution Functions (CDF) of a random variable, encompassing an infinite number of CDF curves. The CDF family of a variable $p$ is expressed as

$$
\mathcal{F}(p) \supseteq \mathcal{F}(p, \boldsymbol{\theta}), \quad \boldsymbol{\theta} \in[\underline{\boldsymbol{\theta}}, \overline{\boldsymbol{\theta}}]
$$

where $\mathcal{F}(p)$ is the CDF curves family, $\boldsymbol{\theta}$ is the distribution coefficients of $p$. The variable $p$ can fall into any one of the parameter categories described in Section 1, according to the involvement of different types of uncertainties. Variables belonging to different categories have different formats of P-boxes, which will be further investigated in Section 3. For the most complex case when both aleatory and epistemic uncertainties are involved, i.e. a Category IV variable, the epistemic uncertainty is presented by the interval $[\underline{\boldsymbol{\theta}}, \overline{\boldsymbol{\theta}}]$. This interval leads to infinite number of CDF curves within the distributional P-box, and this is the reason that a P-box is also known as a uncertainty space of an imprecise probability variable $p$. Fig. 1 illustrates the P-box of a Category IV variable, where the lower and upper bounds of the curve family $\underline{\mathcal{F}}$ and $\overline{\mathcal{F}}$ can be determined by the interval of the distribution coefficients $[\underline{\boldsymbol{\theta}}, \overline{\boldsymbol{\theta}}]$. The shape of a CDF curve (horizontal position and slope) is driven by the mean and variance of a distribution. The horizontal position of the CDF curve is controlled by the mean value; the slope of the CDF curve is controlled by the variance value, i.e. the dispersion degree of the distribution. Based on this principle, the bounds of the P-box have four possibilities as follows:

- The distribution with maximum mean (far right position) and maximum variance (moderate slope);

- The distribution with maximum mean (far right position) and minimum variance (steep slope);

- The distribution with minimum mean (far left position) and maximum variance (moderate slope);

- The distribution with minimum mean (far left position) and minimum variance (steep slope).

The bounds of the P-box can be determined by a simple comparison among the four distributions. Note that, in some cases the P-box bound is not a complete CDF curve of a specific distribution, but a combination of multiple CDF curves. A demonstration to determine bounds of P-boxes with different distribution forms is provided in the first case study (Section 5.2). 


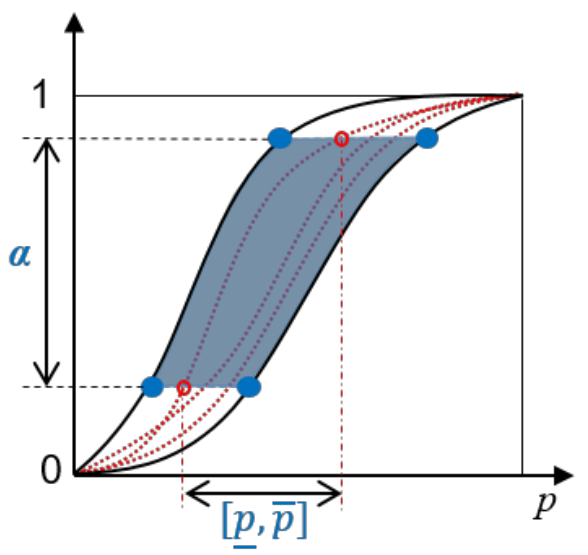

(a) Vertical direction for aleatory uncertainty

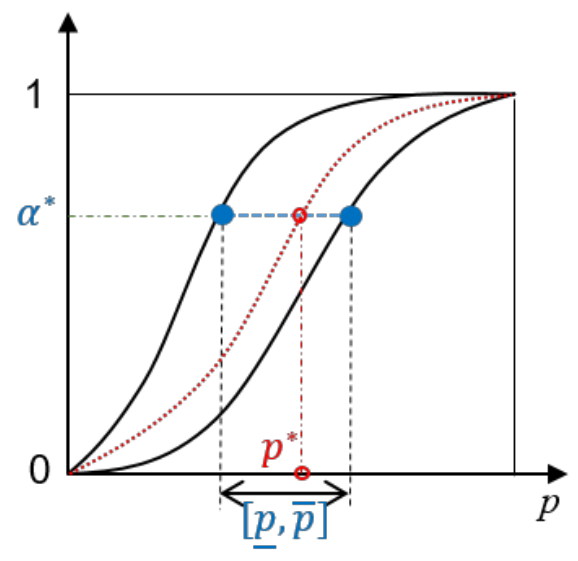

(b) Horizontal direction for epistemic uncertainty

Fig. 1: Investigation of the P-box along two directions

According to the typical categorisation of aleatory and epistemic uncertainties, the aleatory uncertainty refers to the irreducible uncertainty caused by the natural variation of the system; the epistemic uncertainty refers to the reducible uncertainty caused by the lack of knowledge. In stochastic SA, it is important to have a deeper understanding of these two kinds of uncertainties, especially their contributions to the output uncertainty space. This can be achieved through a further investigation of the P-box. As shown in Fig. 1, the P-box is investigated along to two directions, i.e. the vertical and horizontal axes, with different emphases on the aleatory uncertainty and the epistemic uncertainty, respectively.

Fig. 1(a) illustrates the investigation of the P-box along the vertical direction within the overall probability range $[0,1]$. The cumulative probability range $\boldsymbol{\alpha}$ truncated from the vertical axis corresponds to the truncated area within the P-box. Considering the basic hypothesis that the P-box is a set of infinite number of CDF curves, each single CDF curve in the P-box represents a precise probability variable with only aleatory uncertainty. For a single CDF curve in Fig. 1(a), the probability range $\boldsymbol{\alpha}$ corresponds to the variable interval $[\underline{p}, \bar{p}]$ containing only aleatory uncertainty information.

The horizontal investigation of the P-Box focuses on a specific probability value $\alpha^{*}$ within the range $\boldsymbol{\alpha}$, as shown in Fig. 1(b). In the following context, the quantity with superscript * denotes an explicit constant. For a single CDF curve, a fixed probability value $\alpha^{*}$ in the vertical axis corresponds to a fixed variable value $p^{*}$ in the horizontal axis. However, because of the epistemic uncertainty, the CDF family contains infinite number of variable values corresponding to the same probability value $\alpha^{*}$, and thus comprise into the variable interval $[\underline{p}, \bar{p}]$. This interval, different from the one obtained in Fig. 1(a), contains only epistemic uncertainty information.

The above two-direction investigation provides a clear logic to differentiate the contributions of the aleatory and epistemic uncertainties to the P-box of an imprecise probability variable. This investigation is the basis of the two-level procedure in Section 3 to quantify and propagate the uncertainties from the system inputs to the outputs. 


\section{A two-level procedure for uncertainty propagation and quantification}

In this section, a two-level procedure is proposed based on the idea of the two-direction investigation of the P-box in Section 2. The Monte Carlo simulation and optimisation techniques are utilised in the first and second levels, focusing on the aleatory and epistemic uncertainties, respectively. Note that, the analysis of the two kinds of uncertainties cannot be completely separated, but should be performed simultaneously in a uniform framework. The construction of the two levels into a uniform framework is provided in the end of this section.

\subsection{Level I: Monte Carlo simulation for the aleatory uncertainty}

The first level of this overall procedure focuses on the aleatory uncertainty through the Monte Carlo simulation to randomly sample the probability value $\alpha^{*}$ along the vertical direction of the P-box, as shown in Fig. 2. Different categories of parameters have different forms of CDFs/P-boxes, and thus should be analysed separately. As described in Section 1, there are four parameter categories according to the involvement of aleatory and/or epistemic uncertainties. The treatment for each parameter category is explained as follows.

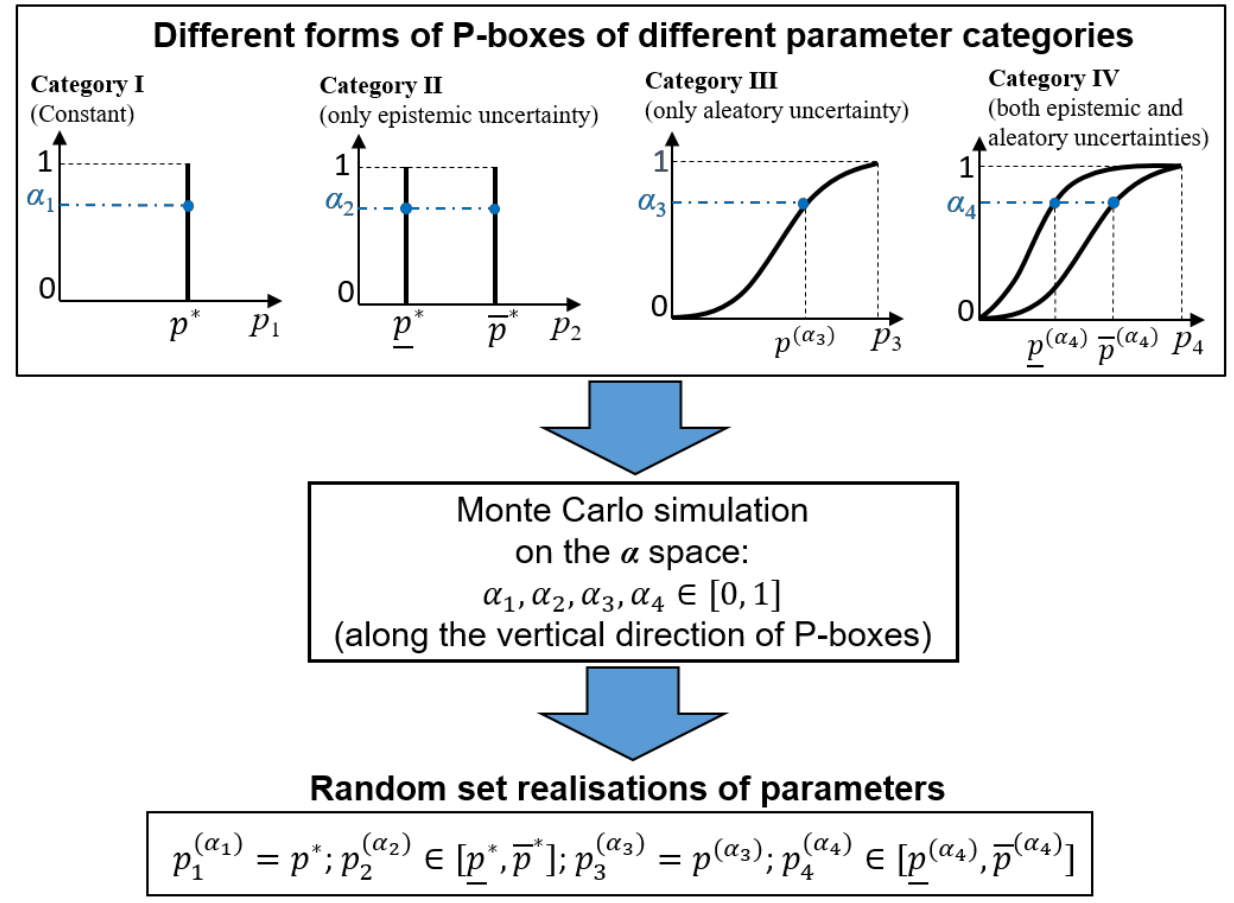

Fig. 2: Monte Carlo simulation for different categories of parameters

Category I): This category of parameter contains no uncertainty. Its CDF appears as an impulse function at the fixed position $p^{*}$, with the amplitude as 1 . During Monte Carlo simulation, for arbitrary probability value $\alpha_{1}$, the corresponding parameter value is always $p^{*}$.

Category II): As an unknown-but-fixed constant fallen within an interval due to the epistemic uncertainty, this kind of parameter has a family of impulse functions, bounded by the given interval $\left[\underline{p}^{*}, \bar{p}^{*}\right]$. The P-box for this kind of parameter appears as a standard rectangle. For randomly sampled value $\alpha_{2}$, the 
corresponding parameter value is no longer a single value, but a range which always equals to the predefined interval $\left[\underline{p}^{*}, \bar{p}^{*}\right]$.

Category III): Involving only aleatory uncertainty, this category of parameter is a variable following a fully determined distribution, and thus it has a single CDF curve. During Monte Carlo simulation, for arbitrary value $\alpha_{3}$, the corresponding parameter value is a constant, however, varying according to the CDF curve. The Categories I and III parameters are not affected by the epistemic uncertainty, and thus these parameters do not need to be updated during a model updating procedure. Nevertheless, the Categories I and III parameters still require appropriate representation and quantification, such that their contributions to the output uncertainty space can be clearly differentiate from that of the epistemic uncertainty.

Category IV): Since both aleatory and epistemic uncertainties are involved, this category of parameters have the normal form P-box as illustrated in Fig. 1. For each sampled probability value $\alpha_{4}$, an interval of the parameter is obtained during the Monte Carlo simulation. Furthermore, bounds of this interval are changing according to different instances of $\alpha_{4}$, because of the P-box with curvilinear bounds as shown in Fig. 2.

In the following context, the term "random set realisation" is utilised to designate the different realisations (i.e. fixed/varying point or interval) of the parameter. The above parameter categorisation provides a clear understanding of different kinds of uncertainties' influences on the resulting random set realisations. Category I represents ideally deterministic parameter whose random set realisation is always a constant point. When aleatory uncertainty is involved, the parameter moves from Category I to Category III, where the random set realisation changes from a fixed point to a changing point, according to different probability values $\alpha$. The epistemic uncertainty renders the random set realisation no long a point, but an interval, by moving the parameter from Category I to Category II. Finally, for Category IV parameter, because of both aleatory and epistemic uncertainties, the resulting random set realisation is an interval with changing bounds according to arbitrary probability values $\alpha$ during Monte Carlo simulation. As the outcome of the first level, the random set realisations will be served as constrains of an optimisation problem in the second level to propagate the uncertainties from the input parameters to the outputs.

\subsection{Level II: Optimisation for the epistemic uncertainty}

The second level is committed to the epistemic uncertainty contained in the random set realisations, and to propagate the epistemic uncertainty from the inputs to the outputs. Here the uncertain system refers to the numerical simulation process containing three key components: input parameters $\boldsymbol{p}$, outputs $\boldsymbol{x}$, and simulator $h(\cdot)$ :

$$
\boldsymbol{x}=h(\boldsymbol{p}) .
$$

The simulator can be a sophisticated finite element model of an engineering structure, or a simple mathematical function extracted from a complex system. The uncertainty propagation is achieved by solving an optimisation problem to determine the minimum and maximum of the outputs, using the random set realisations of the parameters obtained from the first level. The optimisation problem is expressed as: finding 


$$
\min _{\boldsymbol{p}}\{\boldsymbol{x}=h(\boldsymbol{p})\} \text { and } \max _{\boldsymbol{p}}\{\boldsymbol{x}=h(\boldsymbol{p})\},
$$

using the random set realisations as constraints

$$
\left\{\begin{array}{l}
p_{1}^{\left(\alpha_{1}\right)}=p^{*} \\
p_{2}^{\left(\alpha_{2}\right)} \in\left[\underline{p}^{*}, \bar{p}^{*}\right] \\
p_{3}^{\left(\alpha_{3}\right)}=p^{\left(\alpha_{3}\right)} \\
p_{4}^{\left(\alpha_{4}\right)} \in\left[\underline{p}^{\left(\alpha_{4}\right)}, \bar{p}^{\left(\alpha_{4}\right)}\right]
\end{array}\right.
$$

where the superscript * denotes that the value is fixed, the superscript $(\alpha)$ denotes that the value is changing according to the arbitrary $\alpha$, the subscripts " $1-4$ " of $\alpha$ and $p$ denote the categories of the parameters.

Note that, Eq. (4) provides the optimisation problem with simple interval constraints without any complex nonlinear constraint. Furthermore, the interval constraints represent only the epistemic uncertainty, implying the ranges of the intervals are much smaller the whole domain of definition of the parameters in the system. Consequently, the optimisation problem can be solved easily and rapidly by the typical techniques, such as simplex algorithm and interior point method.

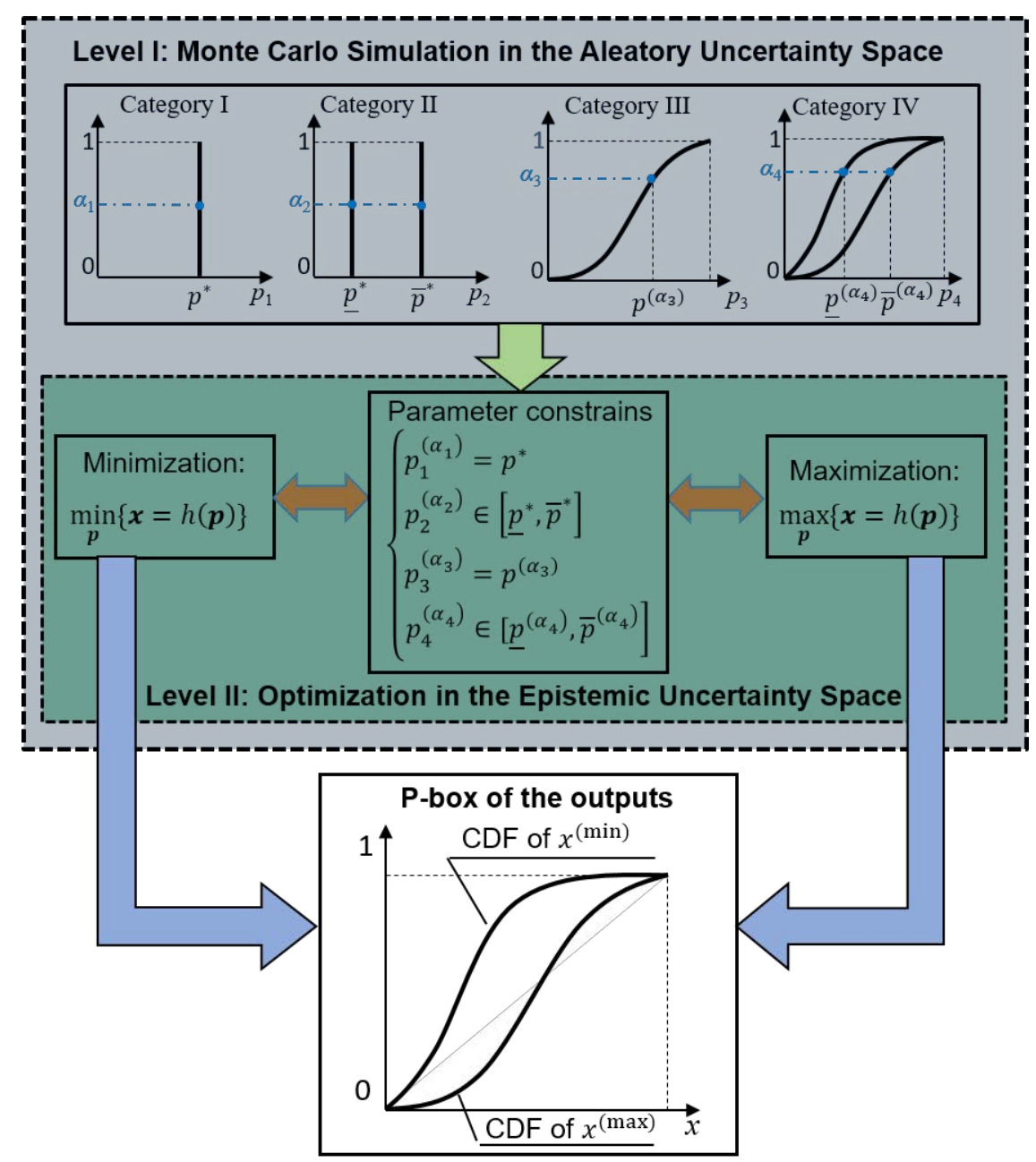

Fig. 3: The two-level procedure for uncertainty quantification and propagation 
The overall two-level procedure for uncertainty quantification and propagation from the inputs to the outputs is illustrated in Fig. 3. Note that, this procedure contains two levels, but not two steps, because the optimisation procedure (Level II) is performed for each sampled point $\boldsymbol{\alpha}$ within the Monte Carlo simulation (Level I). Suppose the sampling size in the Monte Carlo simulation is $N_{\mathrm{MC}}, N_{\mathrm{MC}}$ random set realisations of the input parameters will be obtained and the optimisation is executed $N_{\mathrm{MC}}$ times, generating $N_{\mathrm{MC}}$ pairs of minimum and maximum output values. The CDFs of minimum and maximum outputs are estimated, and the P-box of the output is bounded by these fitted CDFs as shown in the bottom of Fig. 3.

The above two-level approach is significant for stochastic SA, since it provides a clear logic for quantification of both aleatory and epistemic uncertainties, which makes it possible to measure and differentiate the contributions of these two kinds uncertainties to the output uncertainty space.

\section{Sensitivity indices based on the Bhattacharyya distance}

\subsection{Bhattacharyya distance: a quantification metric for the P-box}

The P-box provides a clear representation of the uncertainty space, however, it is still insufficient for SA because of the following reasons:

- SA requires a quantitative measure of the uncertainty space to give an explicit parameter ranking, while the P-box is only a graphic representation;

- The estimation of the CDFs based on the output samples is computationally expensive, making a precise representation of the P-box unpractical for complex systems;

- When multiple outputs are considered in a system, CDFs of the outputs become multi-dimensional, and the construction of the P-box in a multi-dimensional space is out of imagination.

As a result, the Bhattacharyya distance is proposed as a quantification metric for the P-box, which is quantitative, distribution-free, and feasible for more than one output. The basic principle of the Bhattacharyya distance and its application in stochastic model updating have been elaborated in Ref. [1]. Hence, the current work is not focusing on the evaluation method of the Bhattacharyya distance, but a stochastic SA framework where the Bhattacharyya distance can be conveniently implanted.

Fig. 4 illustrates the relationship between the Bhattacharyya distance and the P-box representation of a single output $x$. Instead of using the estimated CDFs of the output samples to construct the P-box, the Bhattacharyya distance is proposed to directly evaluate the overlap between the minimum and maximum output samples. This treatment is executed directly after the two-level procedure in Section 3, where the minimum and maximum output samples are obtained.

The Bhattacharyya distance between two discrete distributions is defined as

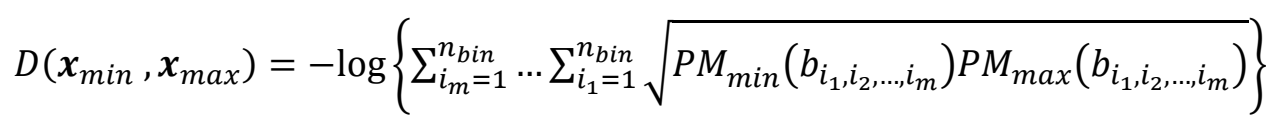

where $\boldsymbol{x}_{\min }$ and $\boldsymbol{x}_{\max }$ are the minimum and maximum samples of the outputs; $m$ is the number of the outputs; $n_{\text {bin }}$ is the number of bins defined for each of the $m$ outputs; $P M_{\mathbf{m}}\left(b_{i_{1}, i_{2}, \ldots, i_{m}}\right)$ is the joint probability mass function (PMF) of the bin $b_{i_{1}, i_{2}, \ldots, i_{m}}$. The bin has $m$ subscripts because it is generated under 
271 a m-dimensional joint-PMF space. The detailed evaluation method of the joint-PMF function can be referred to Ref. [1]. For theoretically completeness, the evaluation procedure is simply recalled as follows.

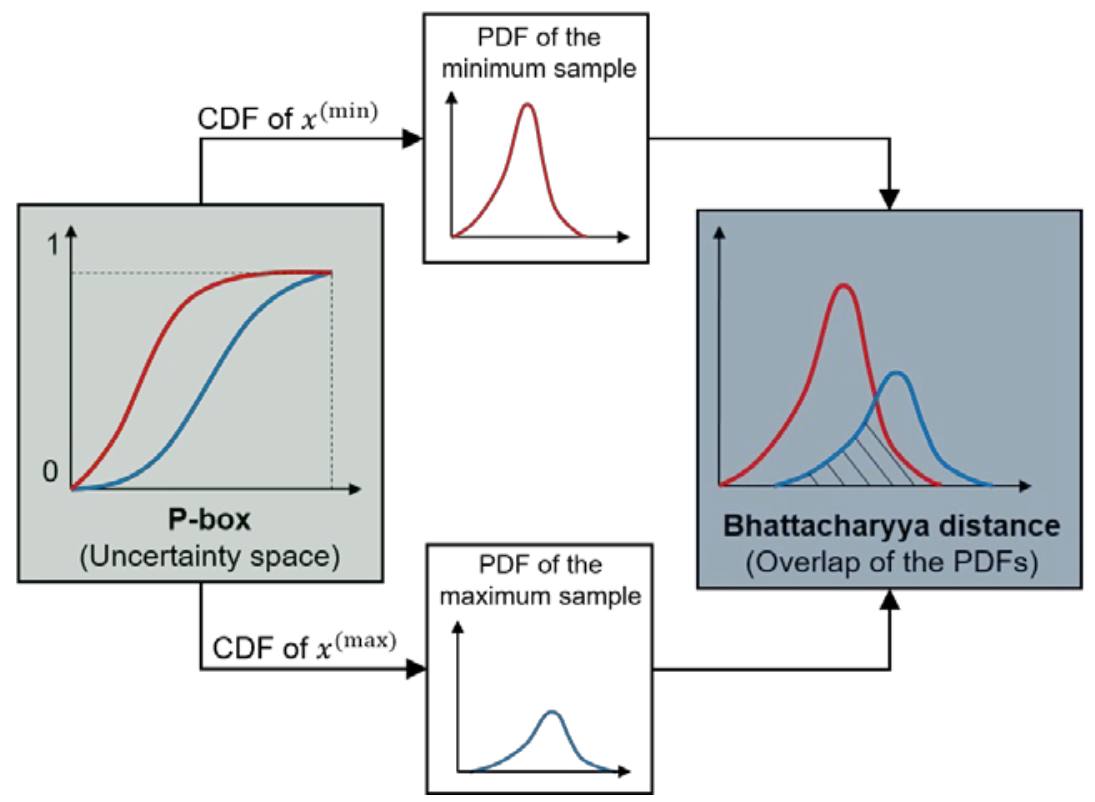

Fig. 4: Relationship between the Bhattacharyya distance and the P-box

1) The output samples $\boldsymbol{x}_{\min }$ and $\boldsymbol{x}_{\max }$ appear as matrices with size as $\boldsymbol{x}_{\mathbf{q}} \in \mathbb{R}^{N \times m}$, where $N$ is the number of data points from Monte Carlo simulation, and $m$ is the number of outputs. Considering the $i$-th columns of the two matrices ( $\boldsymbol{x}_{\min }$ and $\boldsymbol{x}_{\max }$ ), find the lower and upper bounds of all values in both columns. These lower and upper bounds define an interval $I_{i}(\forall i=1, \ldots, m)$ containing all the $i$-th output values in $\boldsymbol{x}_{\min }$ and $\boldsymbol{x}_{\max }$.

2) Within the defined interval $I_{i}$, decide the number of bins $n_{\text {bin }} \cong\left[\frac{N}{10}\right\rceil$, where $[\mathbf{\square}\rceil$ denotes the upper integer of the investigating values;

3) Count the number of points fallen into each bin, i.e. the frequency. Note that, when $m$ is larger than one, the frequency should be counted in a m-dimensional PMF space, and the total number of bins in this joint-PMF space is $N_{b i n}=n_{\text {bin }}{ }^{m}$.

The above procedure is based on the discrete PMF of data samples and thus is applicable to any sample set regardless of its exact distribution format. This makes it especially appropriate for the current application when the sample size after the two-level procedure is too limited to give a precise distribution estimation. Another advantage of the Bhattacharyya distance is that it provides a scalar measure for all the outputs simultaneously, which fulfils the expectation as an elaborate, quantitative, and uniform measure of multiple uncertain outputs.

\subsection{Stochastic sensitivity indices}

The final objective of the stochastic SA, in the background of uncertainty quantification, is described as: Quantify the importance of the input according to how much the uncertainty space of the output can be changed, when the epistemic uncertainty of this input is reduced. This objective is illustrated in Fig. 5 where 
the epistemic uncertainties of the Categories II and IV parameters are completely reduced. For the Category II parameter, the completely reduction of the epistemic uncertainty leads to an impulse function (the red vertical line in the figure). For the Category IV parameter, this will lead to a single CDF curve (the red solid curve in the figure). In the right part of Fig. 5, the change of the output P-box represents the significance of the input parameters. In order to give a quantitative measure, an explicit sensitivity index based on the Bhattacharyya distance is required to rank the significance of each input parameter. Three sensitivity indices, namely the proportional index, the variance-based index, and the comprehensive index, are proposed as follows. Since the reduction of the epistemic uncertainty is executed to either the Category II parameter or to the Category IV parameter, the following three sensitivity indices apply to both Categories II and IV

305 parameters.
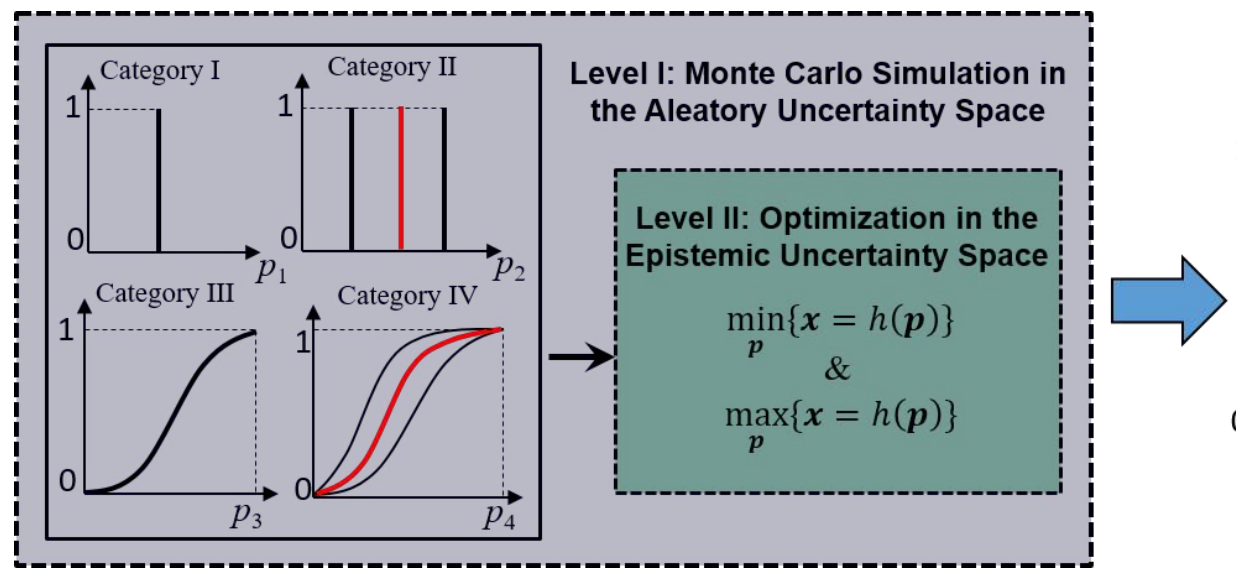

\section{Reduced uncertainty space of the output}

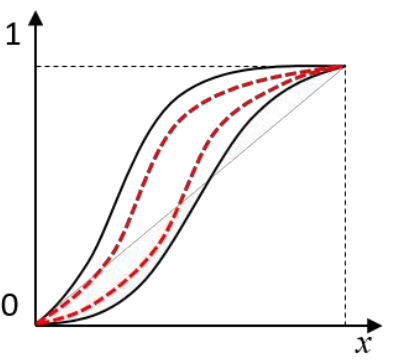

Fig. 5: Reduced output uncertainty space when the epistemic uncertainties of

Categories II and IV parameters are reduced

\section{- The proportional index}

As shown in Fig. 5, the most intuitive manner to measure how much the output uncertainty space is reduced is to calculate the proportion ratio between the reduced uncertainty space and the original one:

$$
S_{i}^{(p r o)}=\frac{D_{0}(\boldsymbol{x})-D_{i}\left(\boldsymbol{x}, p_{i}\right)}{D_{0}(\boldsymbol{x})}
$$

where $S_{i}^{(p r o)}$ is the proportional sensitivity index of the $i$-th input parameter; $D_{0}(\boldsymbol{x})$ is the Bhattacharyya distance of the original uncertainty space of the output; $D_{i}\left(\boldsymbol{x}, p_{i}\right)$ is the Bhattacharyya distance of the output uncertainty space with the epistemic uncertainty of the $i$-th input parameter completely reduced.

Eq. (6) provides a measure of the parameter significance with a direct and simple principle. However, an obvious defect of this equation is that different specified input distributions have different influences on the output uncertainty space. As shown in Fig. 5, there are infinite CDF curves in the P-box of the Category IV parameter, while different curves lead to different output uncertainty spaces. Only randomly investigating one single CDF curve from the input uncertainty space is obviously insufficient. A simple way to deal with this problem is to investigate a series of specified distributions of the parameter, and use the mean of the output uncertainty spaces to replace the single one in Eq. (6). Hence, the improved proportional index is expressed as 
324 where $\mu_{D_{i}}\left(\boldsymbol{x}, p_{i}\right)$ denotes the mean of the multiple Bhattacharyya distance with a series of determined 325 distributions of $p_{i}$. As explained by Eq. (1), the CDF family of input parameter is driven by the interval of its distribution coefficient (e.g. mean or variance) $[\underline{\boldsymbol{\theta}}, \overline{\boldsymbol{\theta}}]$. Hence these different input distributions can be specified by assigning a certain number of equidistant values within the coefficient interval. This treatment is quite sensitive to the number of the coefficients required to describe the P-box. Most distribution types, e.g. Gaussian distribution $N\left(\mu, \sigma^{2}\right)$, uniform distribution $\mathrm{U}(\mathrm{a}, \mathrm{b})$, and Beta distribution Beta(a, $\left.\mathrm{b}\right)$, contain only two distribution coefficients, and thus the full factorial design is feasible to generate a grid of equidistant values. In case of complex distributions with three or even more coefficients, the Design of Experiment (DoE) method is suggested. Considering the typical DoE technique using the orthogonal Latin squares, when there are four coefficients considered and each coefficient contains eight levels, the full factorial design results into $8^{4}=4096$ realisations. In contrast, the orthogonal Latin square design only requires $8^{2}=64$ realisations, which is still the same number as the full factorial design of two-coefficient case. More information of the orthogonal Latin square DoE method can be referred to Ref. [26]. The detailed demonstration for the assignment of specified input distributions can be found in Section 5.3.

In the following context, the number of the specified input distributions is assumed as $N_{\mathrm{sp}}$, and thus $\mu_{D_{i}}\left(\boldsymbol{x}, p_{i}\right)$ in Eq. (7) is estimated from a set of Bhattacharyya distance samples with size as $N_{\text {sp. }}$ The Bhattacharyya distance samples with different sizes lead to different $\mu_{D_{i}}\left(\boldsymbol{x}, p_{i}\right)$, and subsequently, different sensitivity index values. The variation of the index is investigated in the following subsection.

- The variance-based index

The significance of the input parameter is not only reflected by the reduction degree of the output uncertainty space, but also reflected by the dispersion degree of the output uncertainty space when the input P-box is reduced to different single curves. Similar as the above proportional index, a series of specified distributions of $p_{i}$ are utilised, and multiple Bhattacharyya distances are obtained for the corresponding output uncertainty spaces. The dispersion degree of the output uncertainty spaces is measured by the coefficient of variation of the Bhattacharyya distance samples:

$$
S_{i}^{(v a r)}=\frac{\sigma_{D_{i}}\left(\boldsymbol{x}, p_{i}\right)}{\mu_{D_{i}}\left(\boldsymbol{x}, p_{i}\right)}
$$

where the superscript (var) denote the variance-based index; $\sigma_{D_{i}}\left(\boldsymbol{x}, p_{i}\right)$ is the standard deviation of the Bhattacharyya distances with the epistemic uncertainty of $p_{i}$ completely reduced; $\mu_{D_{i}}\left(\boldsymbol{x}, p_{i}\right)$ is the mean of the Bhattacharyya distances. $\sigma_{D_{i}}\left(\boldsymbol{x}, p_{i}\right)$ and $\mu_{D_{i}}\left(\boldsymbol{x}, p_{i}\right)$ are estimated from a set of Bhattacharyya distance samples with size as $N_{\mathrm{sp}}$.

\section{- The comprehensive index}

As discussed above, the proportional index reflects the reduction degree of the output uncertainty; and the variance-based index reflects the dispersion degree of the output uncertainty. Clearly, neither of the 
above indices can give a full measure of the significance of the input according to the overall change of the output uncertainty space. It is reasonable to make a product of these two indices so that a more comprehensive index is obtained as

$$
S_{i}^{(\mathrm{com})}=\frac{D_{0}(\boldsymbol{x})-\mu_{D_{i}}\left(\boldsymbol{x}, p_{i}\right)}{D_{0}(\boldsymbol{x})} \frac{\sigma_{D_{i}}\left(\boldsymbol{x}, p_{i}\right)}{\mu_{D_{i}}\left(\boldsymbol{x}, p_{i}\right)}
$$

where the superscript (com) denotes the comprehensive index. A larger proportional index $S_{i}^{(\text {pro })}$ indicates a stronger reduction effect of the input epistemic uncertainty on the output uncertainty; a larger variancebased index $S_{i}^{(\text {var })}$ indicates a larger variation of the output uncertainty space due to different reduction manners of the input epistemic uncertainty. Consequently, when the comprehensive index $S_{i}^{(\text {com })}$ is larger, the input parameter $p_{i}$ is comprehensively more significant to the output uncertainty space, on both reduction and dispersion effects.

\subsection{Variation of the stochastic sensitivity indices}

A common feature of the stochastic SA methodologies employing Monte Carlo sampling is that the resulting sensitivity index is also stochastic. Specifically, the stochastic feature of the three sensitivity indices in Section 4.2 is derived from two aspects:

1) The evaluation of the Bhattacharyya distance utilises the random samples generated from Monte Carlo simulation. Different random samples lead to slightly different Bhattacharyya distance values.

2) The definition of the sensitivity indices requires not a single specified parameter distribution (i.e. a single CDF curve within a P-box), but a series of specified distributions of the parameter. Different numbers and configurations of the selected CDF curves in the P-box lead to variantion of the sensitivity indices.

Consequently, it is important to investigate the variation feature of the sensitivity indices, especially, when the significances of two parameters are comparable. The bootstrap technique [27] is employed herein to estimate the mean and standard deviation of the stochastic sensitivity indices. Considering the existing sample set of Bhattacharyya distances with size $N_{\text {sp }}$ described in Section 4.2, the basic principle of bootstrap technique in this work is to generate a new sample set (termed as "bootstrap sample set") with the same size as the existing sample set (i.e. $N_{\mathrm{sp}}$ ), using the strategy sampling with replacement. A new value of the sensitivity index can be calculated based on this bootstrap sample set. Assuming the bootstrap process is repeated $N_{\text {bs }}$ times, $N_{\text {bs }}$ bootstrap samples are generated, and subsequently a sample set of the sensitivity index values is obtained with the size as $N_{\mathrm{bs}}$. In the following case studies, the mean of the sensitivity index samples is utilised to rank the significance of the input parameters; the standard deviation of the sensitivity index is provided to assess the variation degree of the index. The smaller the standard deviation, the more precise the index is within the overall stochastic SA approach.

\subsection{Discussion of the calculation cost}

The overall two-level procedure requires an optimisation problem to be solved for each Monte Carlo sample, leading to considerable calculation cost. However, the optimisation problem itself for each sample 
can be solved easily and rapidly using the typical optimisation techniques such as simplex algorithm and interior point method. The simplicity of the optimisation problem is caused by the following reasons.

- The optimisation problem contains only interval constraints of the parameters, without any complex and nonlinear constraint.

- The interval constraints for each Monte Carlo sample are determined in the first level in Section 3, representing only epistemic uncertainty of the P-box. Consequently, the ranges of the interval constraints are much smaller than the whole domain of definition of the parameters in the system.

These interval constraints with small ranges represent an explicit and reduced searching space, which provides convenience when solving the optimisation problem. Even for the complex and strong-nonlinear problem presented in the second case study (Section 6), the optimisation problem can still be solved by the typical interior point method with the CPU calculation time for less than one second in a desk-top computer.

Although the calculation cost for the optimisation is acceptable, in case of complex numerical models (e.g. sophisticated finite element models), the meta-models are suggested as a common rule for Monte Carlo based methodologies. Since each Monte Carlo sampling procedure is independent to each other, the parallel computation [28] can be utilised in this approach to further reduce the calculation time.

\section{Case study I: The Ishigami function}

\subsection{Problem description}

A tutorial case study is given in this section utilising the Ishigami function [29], which is a general example for SA techniques. The Ishigami function is defined as

$$
f(\mathbf{p})=\sin \left(p_{1}\right)+a \sin ^{2}\left(p_{2}\right)+b p_{3}^{4} \sin \left(p_{1}\right),
$$

where $p_{1}, p_{2}$, and $p_{3}$ are the input parameters; $a$ and $b$ are the pre-defined coefficients. In this work, the values of $a$ and $b$ are assigned to be the same as Marrel et al. [30]: $a=7$ and $b=0.1$. The uncertainty characteristic of $p_{1-3}$ is generally set as the uniform distribution on the interval $[-\pi, \pi]$ in the literature. However, this uncertainty characteristic cannot fulfil the application in this work, because the fully determined distribution implies $p_{1-3}$ belong to Category III parameters with only aleatory uncertainty. Considering the objective of the stochastic SA herein, the epistemic uncertainty is the investigating emphasis to assess the uncertainty space of an imprecise distribution, rather than a single CDF curve of a fully determined distribution. As a result, more complex uncertainty characteristics of the parameters are assigned as listed in Table 1. $p_{1}$ and $p_{2}$ are prescribed to follow the uniform distribution, and $p_{3}$ follows the Gaussian distribution. The distribution coefficients, e.g. the bounds, mean, and variance, are not fully determined but fallen into intervals. That is to say, $p_{1-3}$ belong to Category IV parameter in this example.

Table 1: Uncertainty characteristics of the input parameters

\begin{tabular}{ccc}
\hline Category & Parameter Distribution & Epistemic Coefficient \\
\hline IV & $p_{1} \sim U\left(a_{1}, b_{1}\right)$ & $a_{1} \in[-4.0,-3.0] ; b_{1} \in[2.0,3.0]$ \\
IV & $p_{2} \sim U\left(a_{2}, b_{2}\right)$ & $a_{2} \in[-3.0,-1.0] ; b_{2} \in[3.0,5.0]$ \\
IV & $p_{3} \sim N\left(\mu_{3}, \sigma_{3}^{2}\right)$ & $\mu_{3} \in[0.0,1.0] ; \sigma_{3} \in[\sqrt{5}, \sqrt{2}]$ \\
\hline
\end{tabular}


The uncertainty characteristics shown in Table 1 lead to a stochastic SA task different from the normal demonstration on the Ishigami function in the literature, and thus a comparison between the current results and the published results is unavailable. The purpose of this case study is to provide a reproducible result such that a better understanding on the principle of the overall approach is achieved. Detailed assessment and comparison between the results of the proposed approach and the published approaches are presented in the next case study on the NASA UQ Challenge.

\subsection{P-boxes of the inputs and outputs}

The first step of this demonstration is to determine the P-boxes of the input parameters, based on the uncertainty characteristics listed in Table 1 . The P-box of an imprecise uniform distribution can be easily determined by the bounds of the epistemic coefficient intervals. As shown in Fig. 6(a), the P-box of $p_{1}$ is enveloped by the CDFs of $\underline{p}_{1} \sim \mathrm{U}(-4,2)$ and $\bar{p}_{1} \sim \mathrm{U}(-3,3)$. Similarly, the P-box of $p_{2}$ is enveloped by the CDFs of $\underline{p}_{2} \sim \mathrm{U}(-3,3)$ and $\bar{p}_{2} \sim \mathrm{U}(-1,5)$ as shown in Fig. 6(b). The determination of the P-box of $p_{3}$ is more complex than the first two, since the upper or lower bound of the P-box is no longer a complete CDF curve, but a combination of multiple CDF curves as illustrated in Fig. 6(c).

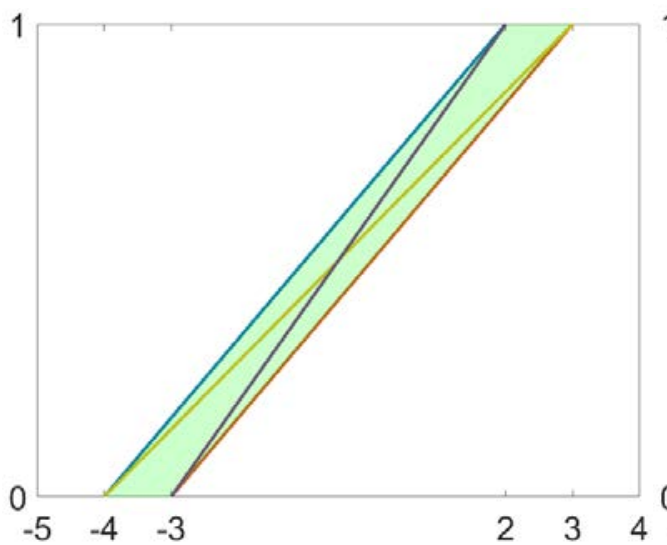

(a) The $1^{\text {st }}$ parameter

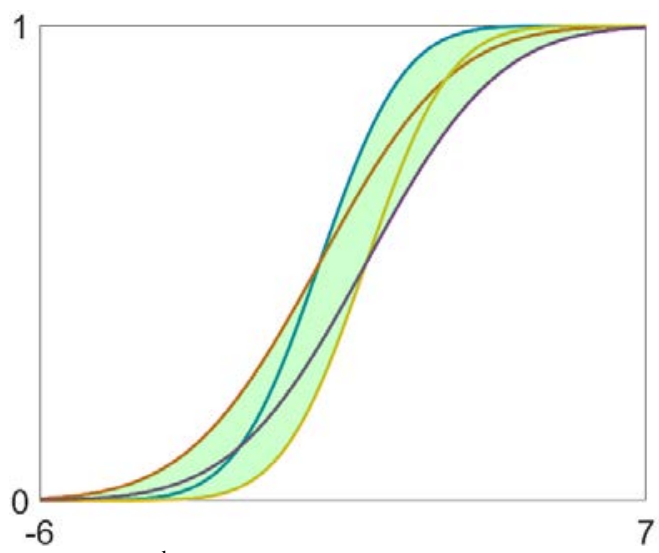

(c) The $3^{\text {rd }}$ parameter

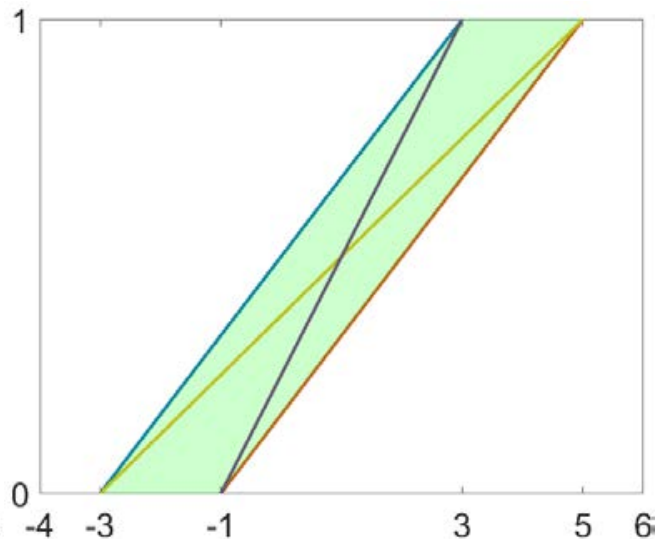

(b) The $2^{\text {nd }}$ parameter

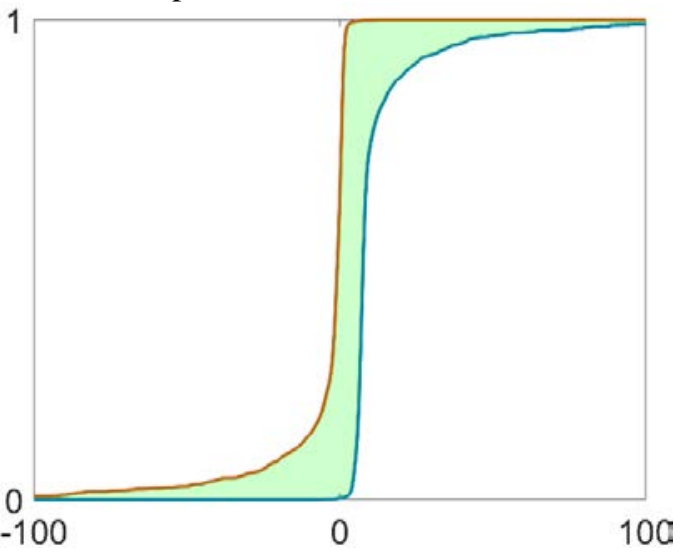

(d) The output

Fig. 6: The P-boxes of the inputs and output variables

As long as the P-boxes of the input parameters are determined, the two-level procedure can be performed to propagate the uncertainty from the inputs to the outputs. In this example, 1000 probability data points 
$445\left(\boldsymbol{\alpha}=\left[\alpha_{1}, \alpha_{2}, \alpha_{3}\right]\right)$ are sampled during the Monte Carlo simulation in the first level. For each sampled $\boldsymbol{\alpha}^{*}$, 446 the intervals of the parameters are determined according to the P-boxes illustrated in Fig. 6(a-c). In fact, for 447 the first two parameters ( $p_{1}$ and $p_{2}$ ), different Monte Carlo samples correspond to the intervals with 448 identical range. For the last parameter $\left(p_{3}\right)$, the range of the interval is changing according to different Monte Carlo samples, because of the curvilinear bounds of the P-box as shown in Fig. 6(c).

After the intervals of the three parameters are determined, an optimisation problem is solved in the second level to find the minimal and maximal values of the output. Since the Monte Carlo sample size is 1000, both the minimal and maximal output sample sets contain 1000 data points. The distributions of the minimal and maximal output samples are estimated using the Kernel Density Estimation (KDE) technique. The CDFs of the minimal and maximal output are utilised to envelop the P-box of the output as illustrated in Fig. 6(d). As described in Section 4.1, the Bhattacharyya distance between the minimal samples and the maximal samples is proposed to provide a quantitative measure of the P-box (i.e. uncertainty space) of the output. During the calculation of the Bhattacharyya value, the number of bins $N_{\text {bin }}$ is taken as 100 , and the calculated distance value is 2.01 .

\subsection{Sensitivity indices based on the Bhattacharyya distance}

After the output uncertainty space has been quantified by the Bhattacharyya distance, the following step focuses on how the uncertainty space of the output can be changed when the epistemic uncertainty of a single input parameter is reduced. Taking $p_{1}$ for example, its epistemic uncertainty is controlled by two epistemic coefficients, i.e. $a_{1}$ and $b_{1}$ in Table 1 . Ten levels of these two coefficients are investigated by assigning ten equidistant values within their intervals, respectively. The full factorial design results in 100 configurations of $a_{1}$ and $b_{1}$, i.e. $N_{\mathrm{sp}}=100$ specific CDF curves within the P-box of $p_{1}$. Because of the simplicity of each optimisation as explained in Section 4.4, the typical interior point method is sufficient to solve the problem. For a complete analysis process of the $1^{\text {st }}$ parameter $p_{1}$, the calculation time is 747.12 s using a desk-top computer with four processors. When the parallel computation is performed at a small scale computer cluster with 36 processors, the calculation time is reduced to $183.60 \mathrm{~s}$.

The two-level procedure is executed for each single curve of $p_{1}$ meanwhile keeping the full P-boxes of $p_{2}$ and $p_{3} .100$ reduced P-boxes of the output are obtained, and accordingly a sample with 100 Bhattacharyya distance values is available. The proportional, variance-based, and comprehensive sensitivity indices of $p_{1}$ are evaluated according to the equations in Section 4.2, and the bootstrap method is utilised to estimate the means and standard deviations of these three indices, respectively. The number of bootstrap samples utilised in this example is $N_{\mathrm{bs}}=1000$. The same strategy is performed for $p_{2}$ and $p_{3}$, and the ranking results according to the means of the bootstrap samples are presented in Table 2, where the superscript denotes the standard deviation of the bootstrap sample. All of the standard deviation values are dramatically smaller than the corresponding mean values, implying the variation of the index is low, and thus the ranking results according to the means of the bootstrap samples are precise.

Clearly, different indices lead to different ranking results. According to the proportional index, $p_{2}$ is the most significant one, implying $p_{2}$ has the largest influence in reducing the output uncertainty space. 
According to the variance-based index, $p_{1}$ is the most significant one, implying different realisation of $p_{1}$ within its epistemic uncertainty space have the largest influence on the variation of the output uncertainty space. The comprehensive index integrating the effects of the first two indicates the significance ranking as $p_{2}>p_{1}>p_{3}$. Note that, the significance ranking can be easily changed by the prescribed intervals of the epistemic coefficients in Table 1 . The case study is presented to provide a tutorial demonstration of the overall stochastic SA approach. Hence, the ranking results in Table 2 are not necessarily to be the same as results in other literature about the Ishigami function.

Table 2: Ranking results of the parameters of the Ishigami function

\begin{tabular}{cccc}
\hline \multirow{2}{*}{ Rank } & \multicolumn{3}{c}{ Results according to different indices } \\
\cline { 2 - 4 } & $S_{i}^{(\text {pro })}$ & $S_{i}^{(\text {var })}$ & $S_{i}^{(\text {com })}$ \\
\hline 1 & $p_{2}(0.9125)^{(0.0017)}$ & $p_{1}(0.2957)^{(0.0278)}$ & $p_{2}(0.1642)^{(0.0107)}$ \\
2 & $p_{1}(0.5027)^{(0.0145)}$ & $p_{3}(0.2891)^{(0.0177)}$ & $p_{1}(0.1490)^{(0.0177)}$ \\
3 & $p_{3}(0.4878)^{(0.0149)}$ & $p_{2}(0.1800)^{(0.0117)}$ & $p_{3}(0.1410)^{(0.0098)}$ \\
\hline
\end{tabular}

\section{Case study II: The NASA UQ challenge problem}

\subsection{Problem description}

Released in 2014, the NASA UQ challenge [23] has been developed as a benchmark problem for uncertainty treatment techniques, containing multiple sub-problems such as uncertainty characterization, SA, uncertainty propagation, and robust design. In this case study, the SA sub-problem is solved to demonstrate performance of the Bhattacharyya distance metric within the proposed stochastic SA approach. Fig. 7 presents the formulation of the SA problem, including the uncertain parameters $\boldsymbol{p}$, outputs $\boldsymbol{x}$, and multiple simulators $h_{i}(\boldsymbol{p})$ given as black-box models. This system contains 21 uncertain parameters, i.e. 4 Category II parameters (with only epistemic uncertainty), 4 Category III parameters (with only aleatory uncertainty), and 13 Category IV parameters (with both epistemic and aleatory uncertainties), whose uncertain characteristics are listed in Table 3. The outputs $\boldsymbol{x} \in \mathbb{R}^{5}$ are evaluated by the multiple black-box models $h$ :

$$
\begin{cases}x_{1}=h_{1}\left(p_{i}\right) & i=1, \cdots, 5 ; \\ x_{2}=h_{2}\left(p_{i}\right) & i=6, \cdots, 10 ; \\ x_{3}=h_{3}\left(p_{i}\right) & i=11, \cdots, 15 ; \\ x_{4}=h_{4}\left(p_{i}\right) & i=16, \cdots, 20 \\ x_{5}=p_{21} . & \end{cases}
$$




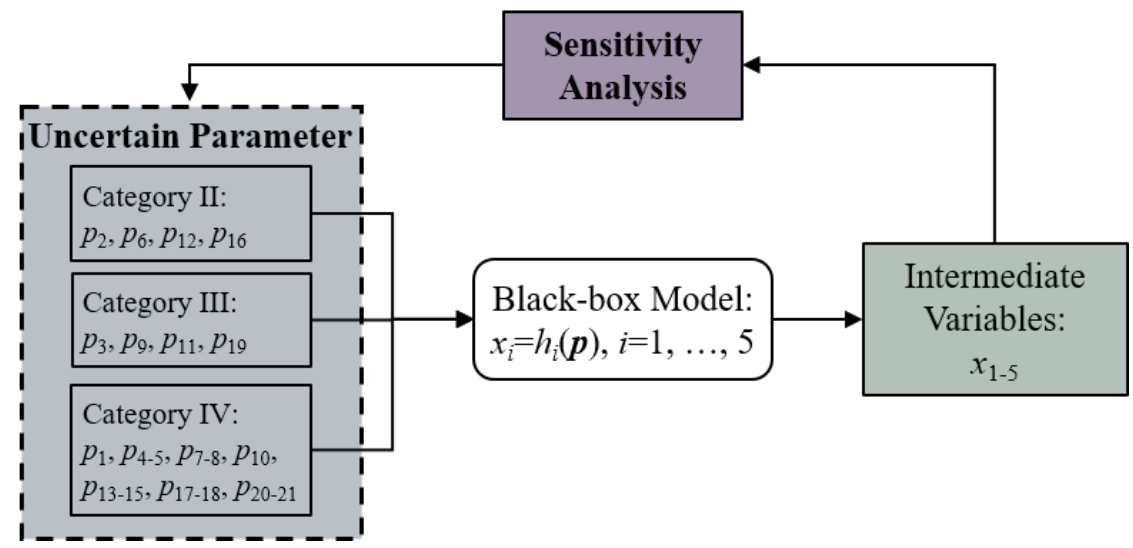

Fig. 7: The formulation and tasks of the SA problem in the NASA UQ challenge

Table 3: The uncertainty characteristics of the 21 input parameters

\begin{tabular}{ccccc}
\hline $\begin{array}{c}\text { Sub- } \\
\text { models }\end{array}$ & Parameter & Category & Distribution & Uncertainty characteristics \\
\hline & $p_{1}$ & IV & Beta & $\mu_{1} \in[0.6,0.8], \sigma_{1}^{2} \in[0.02,0.04]$ \\
$h_{1}$ & $p_{2}$ & II & Constant & $p_{2} \in[0.0,1.0]$ \\
& $p_{3}$ & III & Uniform & $a_{3}=0, b_{3}=1$ \\
& $p_{4}, p_{5}$ & IV & Multiple & $\mu_{i} \in[-5.0,5.0], \sigma_{i}^{2} \in[0.0025,4.0]$ \\
& $p_{6}$ & II & Constant & $\rho \in[-1.0,1.0], i=4,5$ \\
\hline & $p_{7}$ & IV & Beta & $a_{7} \in[0.982,3.537], b_{7} \in[0.619,1.080]$ \\
$h_{2}$ & $p_{8}$ & IV & Beta & $a_{8} \in[7.450,14.093], b_{8} \in[4.285,7.864]$ \\
& $p_{9}$ & III & Uniform & $a_{9}=0, b_{9}=1$ \\
& $p_{10}$ & IV & Beta & $a_{10} \in[1.520,4.513], b_{10} \in[1.536,4.750]$ \\
\hline$h_{11}$ & III & Uniform & $a_{11}=0, b_{11}=1$ \\
& $p_{12}$ & II & Constant & $p_{12} \in[0.0,1.0]$ \\
& $p_{13}$ & IV & Beta & $a_{13} \in[0.412,0.737], b_{13} \in[1.000,2.068]$ \\
& $p_{14}$ & IV & Beta & $a_{14} \in[0.931,2.169], b_{14} \in[1.000,2.407]$ \\
& $p_{15}$ & IV & Beta & $a_{15} \in[5.435,7.095], b_{15} \in[5.287,6.945]$ \\
\hline-- & $p_{16}$ & II & Constant & $p_{16} \in[0.0,1.0]$ \\
& $p_{17}$ & IV & Beta & $a_{17} \in[1.060,1.662], b_{7} \in[1.000,1.488]$ \\
& $p_{18}$ & IV & Beta & $a_{18} \in[1.000,4.266], b_{18} \in[0.553,1.000]$ \\
& $p_{19}$ & III & Uniform & $a_{19}=0, b_{19}=1$ \\
$h_{4}$ & $p_{20}$ & IV & Beta & $a_{20} \in[7.530,13.492], b_{20} \in[4.711,8.148]$ \\
\hline & & IV & Beta & $a_{21} \in[0.421,1.000], b_{21} \in[7.772,29.621]$ \\
\hline & & &
\end{tabular}

509 In the background of uncertainty analysis, the stochastic SA in this example has the task as: For the sub510 model $h_{1}$, rank the 4 Category II and IV parameters (i.e. $p_{1}, p_{2}, p_{4}$, and $p_{5}$,) according to their significance 511 on the uncertainty space of $x_{1}$, when the epistemic uncertainty of the parameter is reduced. And do the same 512 for other sub-models $h_{2}, h_{3}$, and $h_{4}$. 
Differing from the classical SA investigating the variation of unknown-but-fixed constants, the problem herein is established upon the reduction or variation of the uncertainty space of the imprecise random variables. This task involves multiple uncertain parameter categories and multiple distribution formats, which provide a veritable challenge for SA in the uncertainty quantification background. In order to assess the results, three published works on the NASA UQ challenge problem, namely the papers by Patelli et al. [7], Pedroni et al. [24], and McFarland [25], are used as references to compare with the results of the current work.

\subsection{Ranking result of $p_{1}, p_{2}, p_{4}$, and $p_{5}$ according to $x_{1}$}

The SA results for the first output $x_{1}$ are shown in Table 4. The optimisation algorithm used in this example is still the interior point method. The calculation time of a complete analysis process for $p_{1}$ at a four-processor desk-top computer is $4342.08 \mathrm{~s}$. When the parallel computation method is employed at a computer cluster with 36 processors, the calculation time is dramatically reduced to $312.07 \mathrm{~s}$. The $2^{\text {nd }}-4^{\text {th }}$ columns list the results from different references, whose ranking orders are not exactly the same. All these three works get same conclusion that $p_{1}$ and $p_{5}$ are the first two most important parameters. However, the rankings about $p_{4}$ and $p_{2}$ are different. This demonstrates that different approaches using different quantification indices may get different results.

Table 4: The parameter rankings of $p_{1}, p_{2}, p_{4}$, and $p_{5}$ according to $x_{1}$

\begin{tabular}{ccccccc}
\hline \multirow{2}{*}{ Rank } & \multicolumn{3}{c}{ Results in the references } & \multicolumn{3}{c}{ Results in the current work } \\
\cline { 2 - 7 } & Patelli [7] & Pedroni [24] & McFarland [25] & $S_{i}^{(\text {pro })}$ & $S_{i}^{(\text {var })}$ & $S_{i}^{(\text {com })}$ \\
\hline 1 & $p_{1}$ & $p_{1}$ & $p_{1}$ & $p_{1}(0.715)$ & $p_{2}(0.215)$ & $p_{1}(0.137)$ \\
2 & $p_{5}$ & $p_{5}$ & $p_{5}$ & $p_{5}(0.199)$ & $p_{1}(0.192)$ & $p_{5}(0.036)$ \\
3 & $p_{4}$ & $p_{4}$ & $p_{2}$ & $p_{4}(0.166)$ & $p_{5}(0.182)$ & $p_{4}(0.025)$ \\
4 & $p_{2}$ & $p_{2}$ & $p_{4}$ & $p_{2}(0.099)$ & $p_{4}(0.154)$ & $p_{2}(0.021)$ \\
\hline
\end{tabular}

The last three columns of Table 4 present the ranking results of the current work according to three different indices, namely the proportional index $S_{i}^{(\text {pro })}$, the variance-based index $S_{i}^{(v a r)}$, and the comprehensive index $S_{i}^{(\mathrm{com})}$. The index value is provided in parenthesis after each parameter in the table. The ranking result according to the proportional index is the same as the results from the first two references. However, the ranking result according to the variance-based index is different from all of the three results in the references. Nevertheless, the variance-based index values of these four parameters are similar with each other, implying the four parameters have comparable influence on the dispersion degree of the output uncertainty space. This is also the reason that the ranking based on the comprehensive index is the same as the one according to the proportional index. 
Sensitivity results for $x_{2}$ are more complex than that for $x_{1}$, since different referenced works obtain quite different parameter rankings, as shown in Table 5 . The results in the references are different in ranking the 545 last two parameters ( $p_{8}$ and $p_{10}$ ). In the work of McFarland [25], $p_{8}$ and $p_{10}$ were regarded to be negligible, and thus they were not included in McFarland's ranking result.

In Table 5, the differences are also detected among the results according to the three indices in the current work. Using the proportional index, $p_{7}$ is demonstrated as the most significant parameter. However, in the ranking according to the variance-based index, $p_{6}$ is the most significant parameter. By integrally considering both reduction and dispersion effects, the comprehensive index provides the significant ranking as: $p_{7}>p_{6}>p_{8}>p_{10}$. Note that this index shows $p_{7}$ is the most significant parameter, while in the referenced works, $p_{6}$ was regarded as the most significant one.

Table 5: The parameter rankings of $p_{6}, p_{7}, p_{8}$, and $p_{10}$ according to $x_{2}$

\begin{tabular}{ccccccc}
\hline \multirow{2}{*}{ Rank } & \multicolumn{3}{c}{ Results in the references } & \multicolumn{3}{c}{ Results in the current work } \\
\cline { 2 - 7 } & Patelli [7] & Pedroni [24] & McFarland [25] & $S_{i}^{(\text {pro })}$ & $S_{i}^{(\text {var })}$ & $S_{i}^{(\text {com })}$ \\
\hline 1 & $p_{6}$ & $p_{6}$ & $p_{6}$ & $p_{7}(0.643)$ & $p_{6}(0.187)$ & $p_{7}(0.101)$ \\
2 & $p_{7}$ & $p_{7}$ & $p_{7}$ & $p_{6}(0.488)$ & $p_{7}(0.157)$ & $p_{6}(0.091)$ \\
3 & $p_{8}$ & $p_{10}$ & -- & $p_{8}(0.483)$ & $p_{10}(0.144)$ & $P_{8}(0.070)$ \\
4 & $p_{10}$ & $p_{8}$ & -- & $p_{10}(0.458)$ & $p_{8}(0.138)$ & $P_{10}(0.063)$ \\
\hline
\end{tabular}

To explain this difference between the current results and the referenced results, a further investigation is performed for $p_{6}$ and $p_{7}$. Fig. 8 illustrates the samples of the Bhattacharyya distances of the output uncertainty spaces, when the epistemic uncertainties of $p_{6}$ and $p_{7}$ are reduced, respectively. Recall the SA framework in Fig. 5, the P-box of a parameter is reduced into a single CDF curve, meaning its epistemic uncertainty is completely reduced, and subsequently the reduction degree of the output P-box is investigated. However, not only a single specified CDF, but a series of CDFs of this parameter is utilised, leading to a series of changed P-boxes of the output. In this example, the number of the investigating CDFs is 100 . As shown in Table $3, p_{6}$ is a Category II parameter within the interval [0.0, 0.1], and thus 100 values equispaced along this interval are assigned. Differently, $p_{7}$ is a Category IV parameter following imprecise Beta distribution, whose epistemic uncertainty is driven by $a_{7} \in[0.982,3.537]$ and $b_{7} \in[0.619,1.080]$. Hence 10 equidistant values within each interval of $a_{7}$ and $b_{7}$ are respectively assigned. And the full factorial design is performed to specify 100 Beta distributions of $p_{6}$. After these 100 P-boxes of the output are obtained, the Bhattacharyya distance is utilised to quantify the P-boxes, and thus the histograms for $p_{6}$ and $p_{7}$ in Fig. 8 are plotted based on 100 Bhattacharyya distance samples, respectively. 


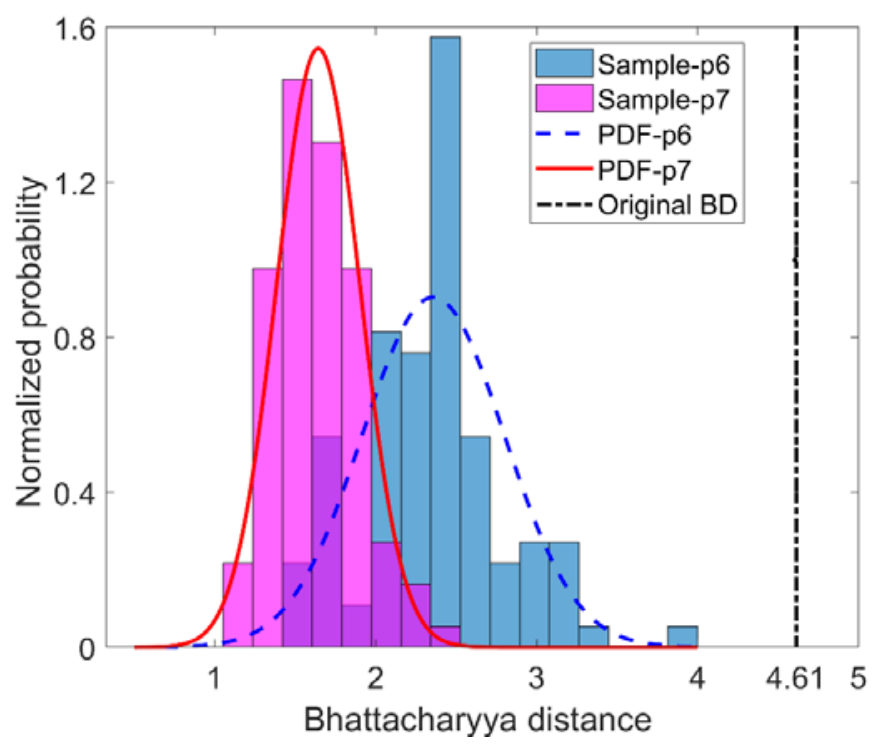

Fig. 8: Comparison of the samples of Bhattacharyya distances for $p_{6}$ and $p_{7}$

In Fig. 8, the Bhattacharyya distance sample according to $p_{6}$ is denominated as "Sample-p6", and the one according to $p_{7}$ is denominated as "Sample-p7". Based on these two samples, two normal distributions are estimated, whose Probability Density Functions (PDFs) are also illustrated in the figure. Note that, the samples are not necessarily following normal distribution. The normal PDFs are presented herein to assist the comparison between these samples. Another important element in Fig. 8 is the dash-dotted vertical line (denominated as "Original BD), denoting the Bhattacharyya distance value of the original P-box of the output $x_{2}$. In other words, when every parameter keeps its epistemic uncertainty, the original output uncertainty space has the Bhattacharyya distance shown by the dash-dotted vertical line (i.e. 4.61). Now Fig. 8 can be investigated from two aspects: the reduction and dispersion of the samples.

1) The reduction of uncertainty spaces is reflected by the distance from the samples to the dash-dotted line. The distance from Sample-p7 to Original BD is larger than that from Sample-p6 to Original BD, implying the output uncertainty space is much more reduced by reducing the epistemic uncertainty of $p_{7}$, rather than $p_{6}$. This coincides with the ranking result with the proportional index in the $5^{\text {th }}$ column of Table 5 , where $p_{7}$ is more significant than $p_{6}$.

2) The dispersion of the uncertainty space is reflected by the variances of the two samples. Sample-p7 (or PDF-p7) is more centralized than Sample-p6 (or PDF-p6), implying various specified CDF of $p_{6}$ have more significant influence on the output uncertainty space. This is why, using the variance-based index, $p_{6}$ is demonstrated to be more important than $p_{7}$ as shown in the $6^{\text {th }}$ column of Table 5.

The above investigation on reduction and dispersion characteristics of the output uncertainty space provides a clear understanding on the significance of $p_{6}$ and $p_{7}$, from different aspects. Consequently, it is suggested to propose the comprehensive index to combine both aspects of the influence and provide an integrated evaluation of the parameters.

The parameter ranking according to $x_{3}$ and $x_{4}$ are listed in Tables 6 and 7, respectively. The results from different references, as well as the results from the current work according to different indices, exhibit more or less differences. However, the situation is the same as the above discussed results for $x_{1}$ and $x_{2}$, and hence 
596

597

598

599

600

601

602

603

604

605

606

607

608

609

610

611

612

613

614

615

616

617

618

619

620

the same investigation procedure can be repeated to analyse the result for $x_{3}$ and $x_{4}$. For clarity, the detailed analysis for $x_{3}$ and $x_{4}$ is omitted.

Table 6: The parameter rankings of $p_{12}, p_{13}, p_{14}$, and $p_{15}$ according to $x_{3}$

\begin{tabular}{ccccccc}
\hline \multirow{2}{*}{ Rank } & \multicolumn{3}{c}{ Results in the references } & \multicolumn{3}{c}{ Results in the current work } \\
\cline { 2 - 7 } & Patelli [7] & Pedroni [24] & McFarland [25] & $S_{i}^{(\text {pro })}$ & $S_{i}^{(\text {var })}$ & $S_{i}^{(\text {com })}$ \\
\hline 1 & $p_{12}$ & $p_{12}$ & $p_{12}$ & $p_{12}(0.944)$ & $p_{12}(1.129)$ & $p_{12}(1.066)$ \\
2 & $p_{15}$ & $p_{13}, p_{15}$ & -- & $p_{14}(0.142)$ & $p_{14}(0.215)$ & $p_{14}(0.030)$ \\
3 & $p_{14}$ & -- & -- & $p_{13}(0.058)$ & $p_{13}(0.085)$ & $p_{13}(0.005)$ \\
4 & $p_{13}$ & $p_{14}$ & -- & $p_{15}(0.458)$ & $p_{15}(0.068)$ & $p_{15}(0.002)$ \\
\hline
\end{tabular}

Table 7: The parameter rankings of $p_{16}, p_{17}, p_{18}$, and $p_{20}$ according to $x_{4}$

\begin{tabular}{ccccccc}
\hline \multirow{2}{*}{ Rank } & \multicolumn{3}{c}{ Results in the references } & \multicolumn{3}{c}{ Results in the current work } \\
\cline { 2 - 7 } & Patelli [7] & Pedroni [24] & McFarland [25] & $S_{i}^{(\text {pro })}$ & $S_{i}^{(\text {var })}$ & $S_{i}^{(\text {com })}$ \\
\hline 1 & $p_{16}$ & $p_{16}$ & $p_{16}$ & $p_{16}(0.737)$ & $p_{18}(0.355)$ & $p_{16}(0.196)$ \\
2 & $p_{18}$ & $p_{18}$ & $p_{18}$ & $p_{18}(0.445)$ & $p_{16}(0.265)$ & $p_{18}(0.158)$ \\
3 & $p_{17}$ & $p_{17}$ & -- & $p_{17}(0.180)$ & $p_{17}(0.185)$ & $p_{17}(0.033)$ \\
4 & $p_{20}$ & $p_{20}$ & -- & $p_{20}(0.019)$ & $p_{20}(0.143)$ & $p_{20}(0.003)$ \\
\hline
\end{tabular}

\section{Conclusions and perspectives}

This work promotes the application of the Bhattacharyya distance as a novel UQ metric in a two-level stochastic SA framework. The two-level procedure provides a clear logic for investigation of the P-box representation, where the aleatory uncertainty is quantified through the Monte Carlo simulation in the first level, and the epistemic uncertainty is propagated from inputs to outputs via optimisation in the second level. The Bhattacharyya distance plays a critical role in constructing the sensitivity indices by providing a quantitative measure of the output uncertainty space. All of the three sensitivity indices are capable of measuring the significance of both Categories II and IV parameters with not only epistemic uncertainty but also aleatory uncertainty, implying this approach is an extension of the deterministic SA which is only feasible for interval sensitivities of the unknown-but-fixed constants (i.e. Category II parameters). The three proposed indices explores different aspects of the sensitivity information of the parameters, that is to say, 1) the proportional index focuses on the reduction effect of the output uncertainty space; 2) the variancebased index focuses on the dispersion degree of the output uncertainty space; and 3) the comprehensive index measures both reduction and dispersion effects of the output uncertainty space. The different information conveyed by different indices is significant for decision makers to achieve a better understanding of the influence of inputs on the outputs from different perspectives.

The tutorial case study utilising the Ishigami function provides a detailed demonstration of the overall approach with reproducible results. In the second case study with the NASA UQ challenge problem, the comparison among the references and current work using different indices exhibits the uniform ranking 
621

622

623

624

625

626

627

628

629

630

631

632

633

tendency, demonstrating the feasibility of the proposed approach. The detailed differences among the ranking results (even in the references themselves) confirms the natural conclusion that different approaches using different sensitivity indices result in different rankings. However, this differences do not necessarily conceal the role of the Bhattacharyya distance as an elaborate, quantitative, and uniform metric for stochastic SA in the background of uncertainty quantification and propagation.

The current work is based on the first-order SA with the assumption that the investigating parameters are independent with each other. Consequently, one of the extensions of this work is a second-order SA investigating the interactions between uncertain parameters. Furthermore, the proposed approach has the potential to be utilised in the case of tail distributions in the context of reliability analysis, with the necessary extension using the directional Monte Carlo sampling techniques, e.g. the subset simulation and Markov chain Monte Carlo algorithm, to achieve a high efficiency for the rare occurrence events.

\section{Acknowledgement}

This is a work supported by the Alexander von Humboldt Foundation, which is greatly appreciated.

\section{Reference}

[1] S. Bi, M. Broggi, M. Beer, The role of the Bhattacharyya distance in stochastic model updating, Mech. Syst. Signal Process. 117 (2019) 437-452. doi:10.1016/j.ymssp.2018.08.017.

[2] S. Bi, S. Prabhu, S. Cogan, S. Atamturktur, Uncertainty Quantification Metrics with Varying Statistical Information in Model Calibration and Validation, AIAA J. 55 (2017) 3570-3583. doi:10.2514/1.J055733.

[3] M. Faes, M. Broggi, E. Patelli, Y. Govers, J. Mottershead, M. Beer, D. Moens, A multivariate interval approach for inverse uncertainty quantification with limited experimental data, Mech. Syst. Signal Process. 118 (2019) 534-548. doi:10.1016/j.ymssp.2018.08.050.

[4] P. Wei, Z. Lu, J. Song, Variable importance analysis: A comprehensive review, Reliab. Eng. Syst. Saf. 142 (2015) 399-432. doi:10.1016/j.ress.2015.05.018.

[5] A. Saltelli, M. Ratto, T. Andres, F. Campolongo, J. Cariboni, D. Gatelli, M. Saisana, S. Tarantola, Global Sensitivity Analysis. The Primer, 2008. doi:10.1002/9780470725184.

[6] I.M. Sobol', Sensitivity Estimates for Nonlinear Mathematical Models, Math. Model. Comput. Exp. 1 (1993) 407-414. doi:1061-7590/93/04407-008.

[7] E. Patelli, D.A. Alvarez, M. Broggi, M. de Angelis, Uncertainty Management in Multidisciplinary Design of Critical Safety Systems, J. Aerosp. Inf. Syst. 12 (2015) 140-169. doi:10.2514/1.I010273.

[8] A.J. Torii, R.H. Lopez, L.F.F. Miguel, Probability of failure sensitivity analysis using polynomial $\begin{array}{llllll}\text { expansion, } & \text { Probabilistic } & \text { Eng. } & \text { (2017) }\end{array}$ doi:10.1016/J.PROBENGMECH.2017.06.001.

[9] Y. Shi, Z. Lu, Z. Li, M. Wu, Cross-covariance based global dynamic sensitivity analysis, Mech. Syst. Signal Process. 100 (2018) 846-862. doi:10.1016/J.YMSSP.2017.08.013.

[10] W. Becker, K. Worden, J. Rowson, Bayesian sensitivity analysis of bifurcating nonlinear models, Mech. Syst. Signal Process. 34 (2013) 57-75. doi:10.1016/j.ymssp.2012.05.010.

[11] S. Bi, Z. Deng, Z. Chen, Stochastic validation of structural FE-models based on hierarchical cluster analysis and advanced Monte Carlo simulation, Finite Elem. Anal. Des. 67 (2013) 22-33. doi:10.1016/j.finel.2012.12.005.

[12] M. Beer, S. Ferson, V. Kreinovich, Imprecise probabilities in engineering analyses, Mech. Syst. Signal Process. 37 (2013) 4-29. doi:10.1016/j.ymssp.2013.01.024.

[13] P. Wei, J. Song, S. Bi, M. Broggi, M. Beer, Z. Lu, Z. Yue, Non-intrusive stochastic analysis with parameterized imprecise probability models: I. Performance estimation, Mech. Syst. Signal Process. 
124 (2019) 349-368. doi:10.1016/J.YMSSP.2019.01.058.

[14] P. Wei, J. Song, S. Bi, M. Broggi, M. Beer, Z. Lu, Z. Yue, Non-intrusive stochastic analysis with parameterized imprecise probability models: II. Reliability and rare events analysis, Mech. Syst. Signal Process. 126 (2019) 227-247. doi:10.1016/J.YMSSP.2019.02.015.

[15] C. Li, S. Mahadevan, Relative contributions of aleatory and epistemic uncertainty sources in time series prediction, Int. J. Fatigue. 82 (2016) 474-486. doi:10.1016/J.IJFATIGUE.2015.09.002.

[16] S. Bi, M. Ouisse, E. Foltête, Probabilistic Approach for Damping Identification Considering Uncertainty in Experimental Modal Analysis, AIAA J. 56 (2018) 4953-4964. doi:10.2514/1.J057432.

[17] S. Ferson, V. Kreinovich, L. Ginzburg, D.S. Myers, K. Sentz, Constructing Probability Boxes and Dempster-Shafer Structures, 2003. doi:10.2172/809606.

[18] R. Schöbi, B. Sudret, Structural reliability analysis for p-boxes using multi-level meta-models, Probabilistic Eng. Mech. 48 (2017) 27-38. doi:10.1016/j.probengmech.2017.04.001.

[19] R. Schöbi, B. Sudret, Uncertainty propagation of p-boxes using sparse polynomial chaos expansions, J. Comput. Phys. 339 (2017) 307-327. doi:10.1016/j.jcp.2017.03.021.

[20] C. Wang, H. Zhang, M. Beer, Computing tight bounds of structural reliability under imprecise probabilistic information, Comput. Struct. $208 \quad$ (2018) 92-104. doi:10.1016/J.COMPSTRUC.2018.07.003.

[21] Scott Ferson, W.T. Tucker, Sensitivity analysis using probability bounding, Reliab. Eng. Syst. Saf. 91 (2006) 1435-1442. doi:10.1016/j.ress.2005.11.052.

[22] D.A. Alvarez, Reduction of uncertainty using sensitivity analysis methods for infinite random sets of indexable type, Int. J. Approx. Reason. 50 (2009) 750-762. doi:10.1016/J.IJAR.2009.02.002.

[23] L.G. Crespo, S.P. Kenny, D.P. Giesy, The NASA Langley Multidisciplinary Uncertainty Quantification Challenge, in: 16th AIAA Non-Deterministic Approaches Conf., 2014: pp. 1-9. doi:10.2514/6.2014-1347.

[24] N. Pedroni, E. Zio, Hybrid Uncertainty and Sensitivity Analysis of the Model of a Twin-Jet Aircraft, J. Aerosp. Inf. Syst. 12 (2015) 73-96. doi:10.2514/1.I010265.

[25] J.M. McFarland, Variance Decomposition for Statistical Quantities of Interest, J. Aerosp. Inf. Syst. 12 (2015) 204-218. doi:10.2514/1.I010261.

[26] Z. Deng, S. Bi, S. Atamturktur, Stochastic model updating using distance discrimination analysis, Chinese J. Aeronaut. 27 (2014) 1188-1198. doi:10.1016/j.cja.2014.08.008.

[27] P. Wei, Z. Lu, X. Yuan, Monte Carlo simulation for moment-independent sensitivity analysis, Reliab. Eng. Syst. Saf. 110 (2013) 60-67. doi:10.1016/J.RESS.2012.09.005.

[28] B. Goller, M. Broggi, A. Calvi, G.I. Schueller, A stochastic model updating technique for complex aerospace structures, Finite Elem. Anal. Des. 47 (2011) 739-752.

[29] T. Ishigami, T. Homma, An importance quantification technique in uncertainty analysis for computer models, in: Proceedings. First Int. Symp. Uncertain. Model. Anal., 1990: pp. 398-403. doi:10.1109/ISUMA.1990.151285.

[30] A. Marrel, B. Iooss, B. Laurent, O. Roustant, Calculations of Sobol indices for the Gaussian process metamodel, Reliab. Eng. Syst. Saf. 94 (2009) 742-751. doi:10.1016/j.ress.2008.07.008. 